\title{
Transmission module partition method based on fuzzy clustering
}

\author{
liqun Zhou ${ }^{1, a}$, Wei Liu ${ }^{1, b}$, Muying Ouyang ${ }^{1, \mathrm{c}}$ \\ ${ }^{1}$ Mechanical Engineering School of Xiangtan University, Xiangtan City 411105, Hunan Province, \\ China \\ aemail: johnzlq@163.com, bemail:156190209@qq.com, cemail:motto1zi@qq.com
}

Keywords: Transmission; module division; fuzzy clustering

\begin{abstract}
The transmission is widely used in various sectors of the important machinery and equipment, In this paper, based on fuzzy clustering analysis, the module of transmission is studied. Construct transmission components function and geometric correlation matrix, corresponding to the fuzzy equivalence matrix, and thus of the transmission were module division. By dividing the module of transmission and improve the efficiency of rapid design, laid the foundation for the different needs of transmission and more rapid response to the market.
\end{abstract}

\section{Introduction}

In the increasingly fierce competition in the market, large-scale custom production requires companies to respond quickly to market, to provide customers with low-cost, personalized products in the shortest time[1]. The product's modular design due to the relatively small number of parts can be used to reduce the cost of the product design, ease of disassembly recycling and maintenance or replacement, and easy to assemble, improving the product reusability and reconstruction. Thus, the modular design has become a mass product ordered most important success factor.

The modular design of the products is divided into two parts, module division and combination, reasonable module partition is the foundation and the key in modular design[2].

In recent years, some scholars at home and abroad have been studied in the field of modular design. $\mathrm{Gu}$ and Sosale[3][4] proposed life cycle oriented modular design methods, using genetic algorithm and simulated annealing algorithm to solve and optimize the model, and the designers of partition scheme, choose the best scheme, so as to realize the modular. National University of Defense Technology Li Guoxi[5] will iterative design topological model and modular combination, and discusses the dynamic modeling method, the function principle of behavior structure, modular design method is proposed based on analysis of the iterative process. Gao Fei[6] and so on from the point of view of product features, Proposed guidelines for functional module division and demand, established evaluation model based on coupling degree program. Zhejiang University Pan Shuangxia[7] taking into account the needs of customers, product assembly and equipment maintenance and other factors, put forward relevant guidelines and evaluation methods of fuzzy clustering based on information entropy. Xiangtan University Zhou Youhang[8], taking into account the complex mechanical product parts from the property, put forward a module partition method based on the important core part . Tang Tao[9] introduces the concept of green design, using the analytic hierarchy process and the green criteria for module division. Transmission as a widely used in automobiles, petrochemicals, agriculture, medicine, food, chemical and other industries of machinery and equipment, study the rapid design system, whether their own development, or the promotion of business interests are of great significance. The modular technology as a core technology to rapidly design, the design of the study transmission has not been molding applications. In this paper, the module partition method based on fuzzy clustering analysis method is adopted to divide the transmission to improve the design efficiency.

\section{Transmission Introduction}

Transmission is by way of a fixed or sub-file to change the output shaft and the input shaft of the transmission gear ratio of the transmission, traditionally based on different ratios, the transmission 
are divided into two categories, A continuously variable transmission and the step transmission. In this paper, a continuously variable transmission(CVT) for the study, mainly comprising 24 parts (see Table 1), a cross-sectional view shown in Figure 1.

Table 1 parts list of continuously variable transmission

\begin{tabular}{|c|c|c|c|}
\hline No & Name & No & Name \\
\hline 1 & Eccentric bearing & 13 & Speed handle \\
\hline 2 & Eccentric wheel & 14 & Small shaft \\
\hline 3 & Input shaft & 15 & Output arm \\
\hline 4 & Speed connecting shaft & 16 & Clutch \\
\hline 5 & Left Speed Bearings & 17 & Output drive shaft \\
\hline 6 & Right Speed Bearings & 18 & Output arm spring \\
\hline 7 & Drive arm & 19 & Output drive gear \\
\hline 8 & Drive arm bearing & 20 & Cover \\
\hline 9 & Drive arm spring & 21 & Apron \\
\hline 10 & Actuator box & 22 & Output shaft \\
\hline 11 & Speed connecting block & 23 & Output gear \\
\hline 12 & Fixed bearing & 24 & Output mechanism box \\
\hline
\end{tabular}

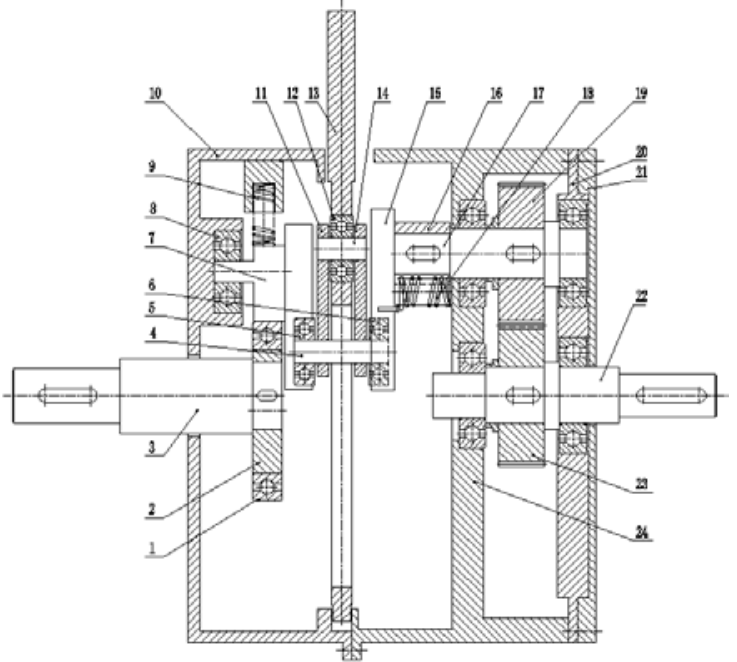

Figure 1 A cross-sectional view of CVT

\section{Module Division of Mathematical Methods}

Divide the product modules, mainly to the function and structure of the product of decomposition, and then by mathematical methods to analyze data decomposition and division. In this paper, we use the function correlation and structure correlation to establish matrix in the process of module division. Then the fuzzy clustering analysis algorithm to analysis and solve the problem.

\section{Fuzzy clustering analysis.}

When using the traditional method of transitive closure, it is needed to establish the fuzzy equivalent matrix on the $\mathrm{X}$. But the disadvantage of this approach is the calculated amount is very large, because often order matrix will be very high. Using the direct clustering method, the amount of calculation will be much smaller, and it is easy to understand. The so-called direct clustering method is a method of fuzzy similarity clustering matrix R directly use, is not necessary to calculate its transitive closure. Principles of its clusters are: $x_{i}$ and $x_{j}$ grade in the horizontal $\Leftrightarrow$ In the fuzzy clustering Figure $\mathrm{R}$ formed in the presence of a significant right of way than or equal to $\lambda$ connection $X_{i}$ and $x_{j}$. Direct clustering methods include two categories, netting and maximum tree method, consistent with their core principles, the difference is netting method is a direct form of clustering method, and the largest tree method is a graphical method of direct clustering method, In contrast, netting method more intuitive and clear.

The so-called netting method is to take a $\lambda$ levels, fuzzy similar matrix $\mathrm{R}$ for $\lambda$ - intercept array $R \lambda$, write symbolic elements in the main diagonal $\lambda$-intercept matrix $R \lambda$. In the lower part of the main diagonal with an asterisk "*" instead of 1 , spaces instead of 0 . And from the "*" the location of the vertical line upward lead, lead horizontal line to the right, through the contact points are connected to each other in order to achieve classification. Now take the domain $X=\{$ I, II, III, $\mathrm{IV}, \mathrm{V}\}$ have similar matrix $\mathrm{R}$ :

$$
R=\left[\begin{array}{ccccc}
1 & 0.88 & 0.68 & 0.24 & 0.12 \\
0.88 & 1 & 0.88 & 0.24 & 0.12 \\
0.68 & 0.88 & 1 & 0.36 & 0.24 \\
0.24 & 0.24 & 0.36 & 1 & 0.72 \\
0.12 & 0.12 & 0.24 & 0.72 & 1
\end{array}\right]
$$

When $0.7<\lambda \leqq 0.8$, get: 


$$
R_{0.7}=\left[\begin{array}{ccccc}
1 & & & & \\
* & 1 & & & \\
& * & 1 & & \\
& & & 1 & \\
& & & * & 1
\end{array}\right]
$$

To analyze the type of netting by an asterisk "*" to the right lead up straight, to give the results shown below. $\{$ I, II, III $\},\{$ IV, V $\}$.

$$
R_{0.7}=\left[\begin{array}{ccccc}
I & & & & \\
* & I I & & & \\
& * & I I I & & \\
& & & I V & \\
& & & * & V
\end{array}\right]
$$

Since the value of $\lambda$ can be within the $[0,1]$ range, when $\lambda$ different values, the matrix $\mathrm{R}$ classification will also change, constituting a different classification by changing the value of $\lambda$ and thus the formation of the elements in between dynamic clustering map, dynamic clustering view of the similarity matrix R shown in Figure 2.

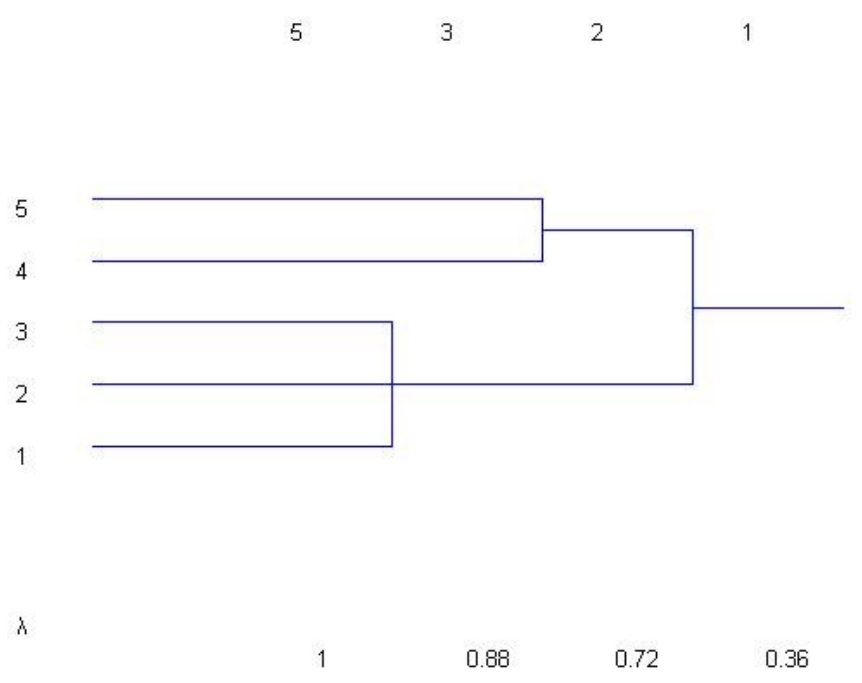

Figure 2 Dynamic clustering map

\section{Correlation Analysis.}

In the module division of the process, only a certain correlation components will be combined in the same module, the paper from the functional correlation and geometric correlation between two aspects to analyze.

Independent function is an important characteristic of a module, in the process of module partition, in order to improve the functional independence of modules, will achieve the same function parts combined together, and that these combinations in between the parts together with correlation function, which is defined as table 2 shows [10].

Table 2 functional definition of the two parts

\begin{tabular}{|l|c|c|}
\hline \multicolumn{1}{|c|}{ RELATIONSHIP } & Relevance & Correlation value \\
\hline To accomplish certain functions, must be used in pairs & Very Strong & 1.0 \\
\hline To accomplish the same function, there is a flow of energy & Strong & 0.8 \\
\hline Presence information flow, have some relevance, interaction & Medium & 0.6 \\
\hline The existence of material flow, moderate association & Weak & 0.4 \\
\hline Functional differences exist essentially independent & Very Weak & 0.2 \\
\hline There is no relationship & None & 0 \\
\hline
\end{tabular}


Geometric relativity refers to the physical connection between parts of the geometry and spatial relations, fastening, verticality, size, parallelism and coaxial degree. Through the contrast analysis, primarily from the connection relation and the geometric relationship accounted for the two important proportion of aspects to consider the geometric correlation between components, which is defined as shown in Table 3.

Table 3 definition of geometric correlation between two parts

\begin{tabular}{|c|c|c|}
\hline RELATIONSHIP & Relevance & Correlation value \\
\hline Tight junctions, is difficult to split, such as welding & Very Strong & 1.0 \\
\hline With strict tolerances, it is difficult to split the connection. & Strong & 0.8 \\
\hline With tolerance requirements, fixed connection, easy to split & Medium & 0.6 \\
\hline With the size of the general requirements, it is easy to split & Weak & 0.4 \\
\hline Contact, but no fit size & Very Weak & 0.2 \\
\hline Not connected & None & 0 \\
\hline
\end{tabular}

\section{Construction of the correlation matrix.}

According to the theory, it can build functional parts respectively associated sub-matrix $A_{\mathrm{m}}$ and geometric correlation sub-matrix $A_{n}$, each relevant matrix by the designer based on the correlation function or geometric definition assessment. Then, the fuzzy relation matrix of components is built according to the related function sub matrix and the geometric sub matrix $A_{\mathrm{n} \times n}$, Using $R_{i j}$ to express the correlation between $i$ and $j$ two parts, and its algorithm is:

$$
R n \times n=\left\{\begin{array}{lc}
\omega_{1} A_{i j}^{m}+\omega_{2} A_{i j}^{n} & (i \neq j) \\
1 & (i=j)
\end{array} \quad(i, j \in\{1,2, \cdots, n\})\right.
$$

In the above formula: $A_{i j}^{m}$-Function relevance between $i$ and $j$;

$A_{i j}^{\mathrm{n}}$ - Geometric relevance between $i$ and $j$;

$\omega_{1}$-Function correlation weighting factor;

$\omega_{2}$ - Geometric correlation weighting factor;

Analytic Hierarchy Process (AHP) or according to the designer's own experience can be obtained for each correlation weighting factor, $\sum_{\mathrm{k}=1}^{n} w_{k}=1$. And fuzzy relationship matrix is symmetric matrix: $R=\left[\begin{array}{cccc}R_{11} & R_{12} & \cdots & R_{1 \mathrm{n}} \\ R_{21} & R_{22} & \cdots & R_{2 n} \\ \vdots & \vdots & \vdots & \vdots \\ R_{n 1} & R_{n 2} & \cdots & R_{n n}\end{array}\right]$

Correlation matrix fuzzy clustering analysis method.

Obtained after the relevant fuzzy relation matrix $\mathrm{R}$ through the above steps, the use of fuzzy cluster analysis fuzzy clustering. Take different thresholds $\lambda$ fuzzy equivalent matrix $\tilde{R}$ interception get different intercept array $R_{\lambda}$, where:

$$
R_{\lambda}(i, j)=\left\{\begin{array}{l}
1, R(i, j) \geq \lambda \\
0, R(i, j)<\lambda
\end{array}\right.
$$

In the same row or column intercept array, the elements of the aggregate value of 1 becomes the same module, others into one another, so that each element is divided into different modules. When $\lambda$ change between 1-0, they will have different classification result, a dynamic clustering module. According to this principle, applied to specific related products, select an appropriate value $\lambda$, will be able to get the last module division scheme. 


\section{CVT module division}

According to the above steps, first of all, to calculate the correlation between the various parts. For example, eccentric wheel bearings and eccentric wheel, in the transfer of power to complete the input function, mutual coordination. So its functional relevance takes 0.8 , geometric correlation takes 1.0. According to equation (1) weighting coefficients $\omega_{1}$ and $\omega_{2}$ respectively, 0.6 and 0.4 . Calculated to obtain the correlation eccentric bearing and the eccentric 0.88 , the correlation can be calculated the same way all the other parts, through the correlation analysis and calculate the fuzzy relation matrix $\mathrm{R}$ :

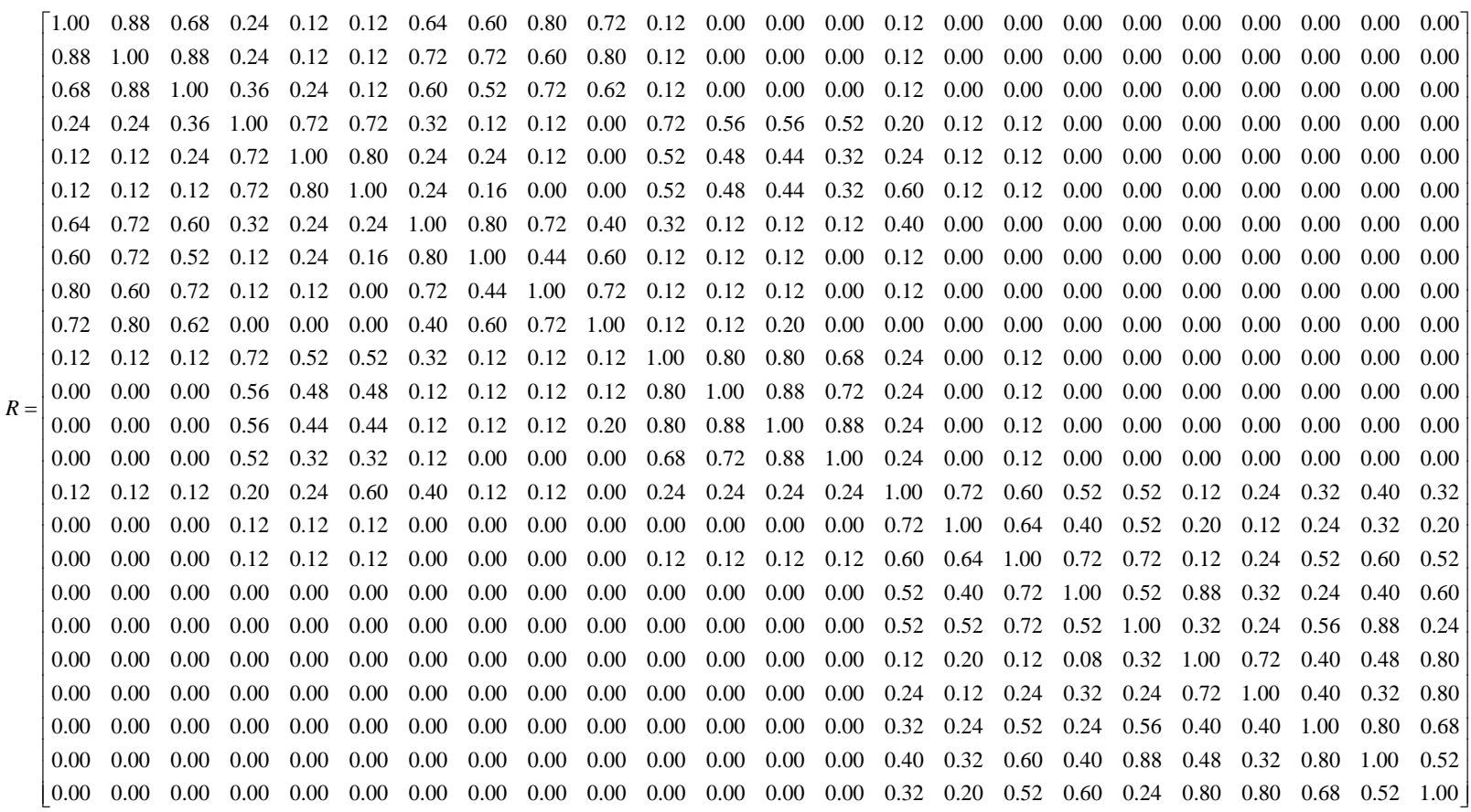

Through the $\lambda$ intercept array analysis, dynamic clustering diagram shown in figure 3 :

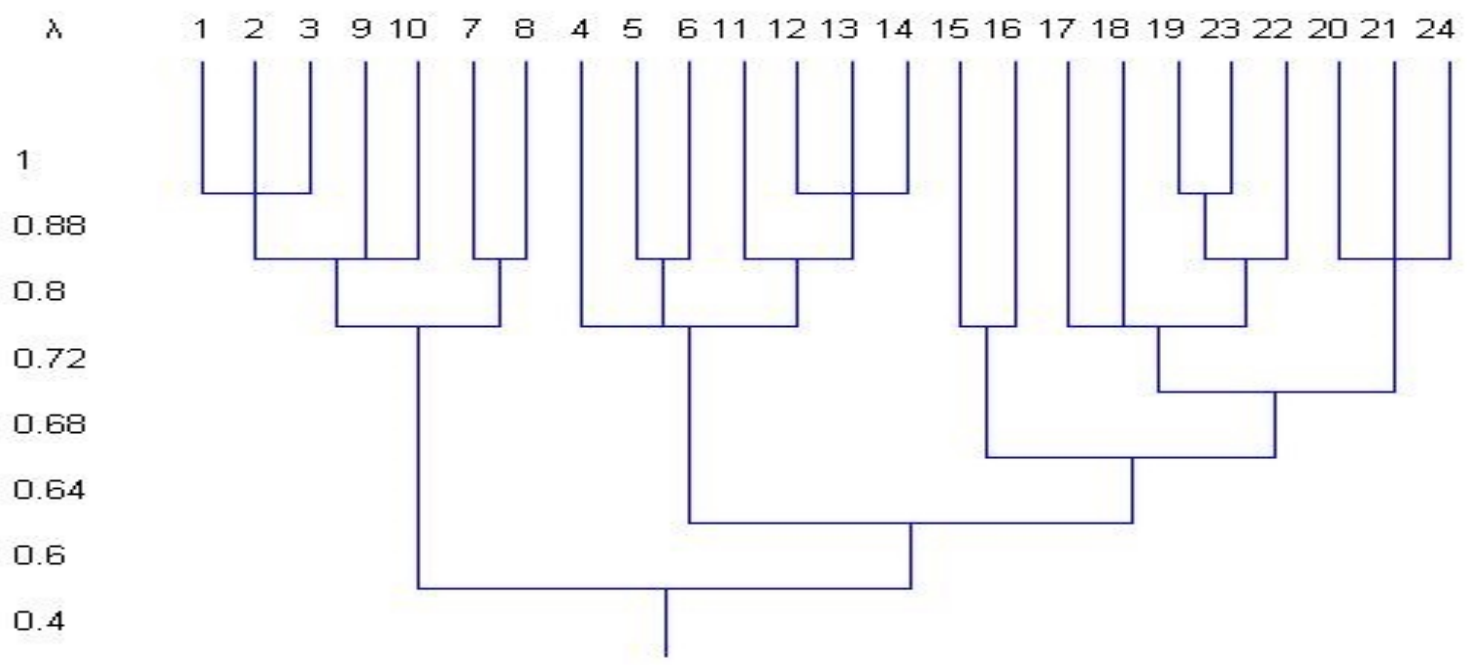

Fig. 3 Dynamic clustering map of CVT module

As can be seen from Figure 3 , when $\lambda>0.72$ the module classification too more, the appropriate $\lambda$ is 0.68 or 0.64 , since the output arm 15 and clutch 16 acts on the output portion of the transmission, into the output of the module. So, take $\lambda=0.64$. The continuously variable transmission is divided into three modules, input part of the module, ratio adjustment section module, the output of the module, broadly consistent with the actual situation. 


\section{Conclusion}

Transmission is an important mechanical equipment widely used in various industries, Based on fuzzy clustering analysis, the paper studies the creation and division of the transmission module. The function of the transmission parts and the correlation matrix of the function of the transmission parts are constructed respectively, and the corresponding fuzzy equivalent matrix is obtained. Threshold $\lambda=0.64$ intercept fuzzy equivalence matrix, the transmission is divided into 3 modules: input module, speed regulation module and output module. Through the division of the module, the design of the transmission is improved, which lays the foundation for the different needs of the market.

\section{Acknowledgment}

Special thanks for open fund of Hunan province department of education (no: 15K129) providing subsidies to this study.

\section{Reference}

[1] Wang Haijun, Sun Baoyuan, Wei Xiaopeng. Modular products based on fuzzy clustering formation process [J]. Computer Integrated Manufacturing Systems -CIMS, 2003, S1:. 123-126.

[2] Wang Rijun, Zhang Jinsheng, Ge Peiqi, Wang Zhi. Classification method based on an integrated module design and fuzzy tree axiom of [J] Agricultural Machinery, 2009,04: 179-183.

[3] GU P,HASHEMIAN M,SOSALE S.An integrated design methodology for life engineering. Ann. CIRP . 1997,46(1):71-4.

[4] GU P,SOSALE S.Product modularization for life engineering. Robotics and Computer Integrated Manufacturing . 1999,15(5):387-401.

[5] Li Guoxi, Wu Jianzhong, Zhang Meng, Gong Jingzhong,Chang Pinyao. Based on function Principle - conduct - Modular structure design method [J]. Journal of the National Defense University, 2009,05: 75-80.

[6] Gao Fei, Xiao Gang, Pan Shuangxia, Chen Jiu Jun, Zhang Yuanming. Product function modules [J]. Journal of Mechanical Engineering, 2007,05: 29-35.

[7] Pan Shuangxia, Gao Fei, Feng Peien. Modular division method of mass customization production mode research [J]. Journal of mechanical engineering, 2003,07:1-6.

[8] Zhou Youhang, Zeng Lei, Zhang Qiao. Classification method based on a modular product core components [J] Machine Design and Research, 2014,02: 1-3 + 7 .

[9] Tang Tao, Liu Zhifeng, Liu Guangfu, Liu Lei. Research on the design method of green modular design [J]. Journal of mechanical engineering, 2003,11:149-154.

[10] Wang Rijun, Zhang Jinsheng, Ge Peiqi, Wang Zhi. Method for design of product module partition method [J]. Journal of Wuhan University of Technology, 2010,01:174-178. 